PIOTR JAWORski (Warszawa)

\title{
A GEOMETRIC POINT OF VIEW ON MEAN-VARIANCE MODELS
}

Abstract. This paper deals with the mathematics of the Markowitz theory of portfolio management. Let $E$ and $V$ be two homogeneous functions defined on $\mathbb{R}^{n}$, the first linear, the other positive definite quadratic. Furthermore let $\Delta$ be a simplex contained in $\mathbb{R}^{n}$ (the set of admissible portfolios), for example $\Delta: x_{1}+\ldots+x_{n}=1, x_{i} \geq 0$. Our goal is to investigate the properties of the restricted mappings $(V, E): \Delta \rightarrow \mathbb{R}^{2}$ (the so called Markowitz mappings) and to classify them. We introduce the notion of a generic model $(\Delta, E, V)$ and investigate the equivalence of such models defined by continuous deformation.

1. Introduction. The portfolio selection theory founded by Markowitz half a century ago $([4,5])$ became a classical part of the modern mathematical finance ([7]) and is a starting point of both practical (see for example $[1,6]$ ) and theoretical courses (for example [2]). The aim of the present paper is to get a better insight into the mathematics lying behind the Markowitz model.

In portfolio theory (see $[5,2,7]$ ) we consider the future rates of return of several financial assets. We model these rates as random variables $r_{i}$, $i=1, \ldots, n$. If $x=\left(x_{1}, \ldots, x_{n}\right)$ denotes a portfolio, i.e. $x_{i}$ is an amount invested in the $i$ th asset, then the associated income (gain, return) is modeled by a random variable

$$
R(x)=\sum_{i=1}^{n} r_{i} x_{i} .
$$

Our goal is to compare the expected return (i.e. the mean) $E(R(x))$ and the variance $V(R(x))$ for all admissible portfolios $x$. We assume that our port$90 \mathrm{C} 20$.

2000 Mathematics Subject Classification: Primary 91B28; Secondary 58K30, 14P99,

Key words and phrases: portfolio theory, Markowitz model. 
folios have the same fixed initial value. Therefore the admissible portfolios are contained in an affine subspace $H \subset \mathbb{R}^{n}$ given by

$$
x_{1}+\ldots+x_{n}=v>0 .
$$

Usually it is assumed that the value of a portfolio equals 1 unit $(v=1)$. Then $R(x)$ becomes the rate of return of the portfolio. Sometimes it is convenient to add some more restrictions. For example if short selling is not allowed we have to assume that all $x_{i}$ are nonnegative. In this case the admissible portfolios are contained in the $(n-1)$-dimensional simplex $\Delta \subset \mathbb{R}^{n}$ defined by

$$
x_{1}+\ldots+x_{n}=1>0, \quad x_{1} \geq 0, \ldots, x_{n} \geq 0 .
$$

Note that $E(R(x))$ is a linear function of $x$ :

$$
E(R(x))=\sum_{i=1}^{n} x_{i} E\left(r_{i}\right)
$$

and $V(R(x))$ is quadratic:

$$
V(R(x))=\sum_{i=1}^{n} x_{i}^{2} V\left(r_{i}\right)+2 \sum_{i<j} x_{i} x_{j} \operatorname{cov}\left(r_{i}, r_{j}\right),
$$

where $\operatorname{cov}\left(r_{i}, r_{j}\right)$ stands for the covariance.

The admissible portfolio $x$ is considered to be effective if it simultaneously maximizes $E(R(x))$ and minimizes $V(R(x))$. In more detail: the admissible portfolio $x$ is effective if for any admissible portfolio $y$,

$$
\begin{aligned}
E(R(y))<E(R(x)) & \vee V(R(y))>V(R(x)) \\
& \vee[E(R(y))=E(R(x)) \wedge V(R(y))=V(R(x))] .
\end{aligned}
$$

Such portfolios are contained in the set of portfolios of relatively minimal variance which are of special interest (see Theorem 3.2).

We introduce the notion of a generic model $(\Delta, E, V)$ (see $\S 4)$ in such a way that two generic models which are close enough to each other have similar polygonal lines of portfolios of relatively minimal variance, and the set of nongeneric models is a proper algebraic subset of the set of all models.

Furthermore we introduce the equivalence of generic triples. We say that $\left(\Delta_{0}, E_{0}, V_{0}\right)$ is equivalent to $\left(\Delta_{1}, E_{1}, V_{1}\right)$ if there exists a continuous family of generic triples $\left(\Delta_{t}, E_{t}, V_{t}\right), t \in[0,1]$, joining them.

We show that equivalent triples have similar polygonal lines of portfolios of relatively minimal variance. Moreover we describe the numerical invariants of the above equivalence.

Basing on this we give a complete classification of generic models describing the three assets case. We show that up to equivalence there are seven possibilities (i.e. there are seven stable shapes of polygonal lines of portfolios of relatively minimal variance). 
Next we study the one-factor models (Sharpe-Lintner models), the simplest models in APT (arbitrage pricing theory) or CAPM (capital asset pricing model) - see $[7, \S \S 8.2,8.7]$. We describe the polygonal line of portfolios of relatively minimal variance for such models. Since models obtained by studying "real" markets are often very close to one-factor models, this gives some insight into the "shape" of the image of a "real life" Markowitz mapping.

This paper is rather technical. Its aim is to provide tools for further more practical studies, like for example:

- constructing Markovitz models with prescribed properties,

- testing the volatility of the covariance matrix of financial returns,

- testing the conjecture of the existence of some dominant factors on a given market.

2. Notation. We use the matrix notation. All vectors are column vectors and the superscript " $T$ " denotes transposition.

The $n$-dimensional mean-variance model is a triple $(\Delta, E, V)$, where:

- $\Delta$ is a simplex in $\mathbb{R}^{n}$ such that the affine space $H$ spanned by all the vertices of $\Delta$ is a hypersurface not containing the origin,

$$
H: \quad h^{T} x=1, \quad h \in \mathbb{R}^{n} ;
$$

- $E$ is a linear function (called mean) on $\mathbb{R}^{n}$,

$$
E(x)=\mu^{T} x, \quad \mu \in \mathbb{R}^{n}, \mu \neq 0 ;
$$

- $V$ is a positive definite quadratic function (called variance) on $\mathbb{R}^{n}$,

$$
V(x)=x^{T} C x, \quad \forall x \neq 0 \quad V(x)>0,
$$

where $C$ is a symmetric positive definite $n \times n$ matrix.

We denote by $\Sigma$ the critical set of the restricted mapping

$$
(V, E): H \rightarrow \mathbb{R}^{2} \text {. }
$$

REMARK. If $E$ is not constant on $H$ then $\Sigma$ coincides with the set of portfolios of relatively minimal variance

$$
\Sigma=\{x \in H: V(x)=\min \{V(z): z \in H \wedge E(z)=E(x)\}\} .
$$

Therefore, for any nonempty subset $K$ of $H$, we denote by $\Sigma_{K}$ the subset of $K$ consisting of portfolios of relatively minimal variance (in $K$ ):

$$
\Sigma_{K}=\{x \in K: V(x)=\min \{V(z): z \in K \wedge E(z)=E(x)\}\} .
$$

We denote by $X_{K}$ the right inverse of the mean $E$ restricted to $\Sigma_{K}$ :

$$
X_{K}: E(K) \rightarrow \Sigma_{K}, \quad E\left(X_{K}(t)\right)=t .
$$

In Section 3 we discuss the uniqueness of $X_{K}$. 
Furthermore we denote by $K^{\perp}$ the linear space consisting of vectors $C$-orthogonal to the affine space spanned by $K$ :

$$
K^{\perp}=\left\{x \in \mathbb{R}^{n}: \forall z_{1}, z_{2} \in K \quad x^{T} C\left(z_{1}-z_{2}\right)=0\right\} ;
$$

moreover, Int $K$ is the interior of $K$ considered as a subset of the affine space spanned by $K$, and $K_{1}+K_{2}$ is the set of sums of vectors from $K_{1}$ and $K_{2}$,

$$
K_{1}+K_{2}=\left\{x+z: x \in K_{1} \wedge z \in K_{2}\right\} .
$$

If $K$ consists of just one point then $K^{\perp}=\mathbb{R}^{n}$ and Int $K=K$.

Next, for any finite set of points of $\mathbb{R}^{n}$, say $z_{1}, \ldots, z_{k}$, we denote by $\Delta\left(z_{1}, \ldots, z_{k}\right)$ the convex polyhedron spanned by the $z_{i}$ 's, i.e.

$\Delta\left(z_{1}, \ldots, z_{k}\right)$

$$
=\left\{z \in \mathbb{R}^{n}: z=a_{1} z_{1}+\ldots+a_{k} z_{k}, a_{i} \in \mathbb{R}, a_{i} \geq 0, a_{1}+\ldots+a_{k}=1\right\} ;
$$

by $H\left(z_{1}, \ldots, z_{k}\right)$ the affine space spanned by the $z_{i}$ 's:

$H\left(z_{1}, \ldots, z_{k}\right)=\left\{z \in \mathbb{R}^{n}: z=a_{1} z_{1}+\ldots+a_{k} z_{k}, a_{i} \in \mathbb{R}, a_{1}+\ldots+a_{k}=1\right\} ;$

and by $\operatorname{Lin}\left(z_{1}, \ldots, z_{k}\right)$ the linear space spanned by the $z_{i}$ 's:

$$
\operatorname{Lin}\left(z_{1}, \ldots, z_{k}\right)=\left\{z \in \mathbb{R}^{n}: z=a_{1} z_{1}+\ldots+a_{k} z_{k}, a_{i} \in \mathbb{R}\right\} .
$$

If $k \leq n$ and $z_{1}, \ldots, z_{k}$ are in general position then $\Delta\left(z_{1}, \ldots, z_{k}\right)$ is a $(k-1)$-dimensional simplex, $H\left(z_{1}, \ldots, z_{k}\right)$ is a $(k-1)$-dimensional space and $\operatorname{Lin}\left(z_{1}, \ldots, z_{k}\right)$ a $k$-dimensional space.

3. Normal forms. Following the standard notation we put

$$
\begin{array}{rlrl}
\alpha=\mu^{T} C^{-1} \mu, & \gamma & =h^{T} C^{-1} h, \\
\beta & =h^{T} C^{-1} \mu, & \delta & =\alpha \gamma-\beta^{2} .
\end{array}
$$

Since $C$ is positive definite, it is invertible and its inverse is also positive definite. Therefore $\alpha$ and $\gamma$ are positive. If $E$ is not constant on $H$ then $\delta$ being the Gram determinant of the $C^{-1}$-product of $h$ and $\mu$ is also positive.

Let $C^{*}$ be the $(n+2) \times(n+2)$ matrix obtained from $C$ by adding two rows and two columns:

$$
C^{*}=\left(\begin{array}{ccc}
0 & 0 & h^{T} \\
0 & 0 & \mu^{T} \\
h & \mu & C
\end{array}\right) .
$$

Lemma 3.1. $\operatorname{det} C^{*}=\delta \operatorname{det} C$.

Proof. We observe that

$$
\left(\begin{array}{ccc}
0 & 0 & h^{T} \\
0 & 0 & \mu^{T} \\
h & \mu & C
\end{array}\right)\left(\begin{array}{ccc}
-1 & 0 & 0 \\
0 & -1 & 0 \\
C^{-1} h & C^{-1} \mu & \mathrm{Id}
\end{array}\right)
$$




$$
\begin{aligned}
& =\left(\begin{array}{ccc}
h^{T} C^{-1} h & h^{T} C^{-1} \mu & h^{T} \\
\mu^{T} C^{-1} h & \mu^{T} C^{-1} \mu & \mu^{T} \\
-h+C C^{-1} h & -\mu+C C^{-1} \mu & C
\end{array}\right) \\
& =\left(\begin{array}{ccc}
h^{T} C^{-1} h & h^{T} C^{-1} \mu & h^{T} \\
\mu^{T} C^{-1} h & \mu^{T} C^{-1} \mu & \mu^{T} \\
0 & 0 & C
\end{array}\right) .
\end{aligned}
$$

Thus

$$
\operatorname{det} C^{*}=\operatorname{det}\left(\begin{array}{cc}
h^{T} C^{-1} h & h^{T} C^{-1} \mu \\
\mu^{T} C^{-1} h & \mu^{T} C^{-1} \mu
\end{array}\right) \cdot \operatorname{det} C=\delta \operatorname{det} C .
$$

THEOREM 3.1. If $n \geq 2$ and $E$ is not constant on $H$ then there exists a coordinate system $y_{0}, y_{1}, \ldots, y_{n-1}$ in $\mathbb{R}^{n}$ such that:

- $V(x)=y_{0}^{2}+y_{1}^{2}+\ldots+y_{n-1}^{2}$;

- $H=\left\{y_{0}=1 / \sqrt{\gamma}\right\}$;

- for $x \in H$,

$$
E(x)=\sqrt{\frac{\delta}{\gamma}} y_{1}+\frac{\beta}{\gamma} .
$$

Proof. We apply the Gram-Schmidt orthonormalization to the vectors

$$
\widehat{v}_{0}=C^{-1} h, \quad \widehat{v}_{1}=C^{-1} \mu,
$$

extended to a basis $\widehat{v}_{0}, \widehat{v}_{1}, \ldots, \widehat{v}_{n-1}$ of $\mathbb{R}^{n}$. We obtain an orthonormal basis $v_{0}, v_{1}, \ldots, v_{n-1}$ for the bilinear product defined by $C$. Moreover

$$
v_{0}=\frac{1}{\sqrt{\gamma}} C^{-1} h, \quad v_{1}=\frac{\gamma C^{-1} \mu-\beta C^{-1} h}{\sqrt{\gamma \delta}} .
$$

The new coordinates $y_{i}$ are the coefficients of vectors in the basis $v_{0}, \ldots, v_{n-1}$, i.e.

$$
x=y_{0} v_{0}+y_{1} v_{1}+\ldots+y_{n-1} v_{n-1} .
$$

Obviously, since the $v_{i}$ 's are orthonormal,

$$
x^{T} C x=\sum_{i=0}^{n-1} y_{i}^{2} .
$$

Furthermore

$$
\begin{aligned}
& x^{T} h=x^{T}\left(\sqrt{\gamma} C v_{0}\right)=\sqrt{\gamma} x^{T} C v_{0}=\sqrt{\gamma} y_{0}, \\
& x^{T} \mu=x^{T} \frac{\sqrt{\gamma \delta} C v_{1}+\beta h}{\gamma}=\sqrt{\frac{\delta}{\gamma}} y_{1}+\frac{\beta}{\gamma} x^{T} h .
\end{aligned}
$$


Therefore, if $x \in H$, i.e. $x^{T} h=1$, then

$$
x^{T} \mu=\sqrt{\frac{\delta}{\gamma}} y_{1}+\frac{\beta}{\gamma} .
$$

COROLlary 3.1. Under the assumption of Theorem 3.1 the critical set $\Sigma$ is a line parallel to the $y_{1}$-axis:

$$
y_{0}=1 / \sqrt{\gamma}, \quad y_{2}=\ldots=y_{n-1}=0,
$$

i.e. it is a shifted line $\operatorname{Lin}\left(v_{1}\right)$,

$$
\Sigma=\left\{\frac{1}{\sqrt{\gamma}} v_{0}\right\}+\operatorname{Lin}\left(v_{1}\right)=H \cap \operatorname{Lin}\left(v_{0}, v_{1}\right) .
$$

Moreover $\Sigma$ is the image of the linear function

$$
X_{H}: \mathbb{R} \rightarrow \Sigma, \quad X_{H}(t)=\frac{1}{\sqrt{\gamma}} v_{0}+\left(t-\frac{\beta}{\gamma}\right) \frac{\sqrt{\gamma}}{\sqrt{\delta}} v_{1} .
$$

Proof. In the $y$-coordinates the hyperplane $H$ is described by $y_{0}=1 / \sqrt{\gamma}$, and the hyperplanes $E(z)=$ const by $y_{1}=$ const. Therefore the relative minimum of $V(z)$, i.e. of the sum of the squares of the $y_{i}$ 's, is attained when $y_{2}=\ldots=y_{n-1}=0$. Thus

$$
\Sigma=\left\{z=\frac{1}{\sqrt{\gamma}} v_{0}+y_{1} v_{1}: y_{1} \in \mathbb{R}\right\}=\left\{\frac{1}{\sqrt{\gamma}} v_{0}\right\}+\operatorname{Lin}\left(v_{1}\right) .
$$

The formula for $X_{H}$ follows from the condition $E\left(X_{H}(t)\right)=t$.

Note:

$$
V\left(X_{H}(t)\right)=\frac{1}{\gamma}+\frac{\gamma}{\delta}\left(t-\frac{\beta}{\gamma}\right)^{2} .
$$

REMARK. Since the critical set $\Sigma$ is a line it is called the critical line.

Let $H_{1}$ be an affine subspace of $H$.

COROLlary 3.2. Under the assumption of Theorem 3.1,

$$
\Sigma_{H_{1}}=H_{1} \cap \Pi \text {, where } \Pi=\operatorname{Lin}\left(v_{1}\right)+H_{1}^{\perp} .
$$

Proof. We shall base on the fact that in the $y$-coordinates the scalar product induced by $C$ is the canonical euclidean scalar product of vectors.

Let $L$ be any affine subspace of $H$, and $z_{1}$ the point of $L$ nearest to the origin. Obviously $z_{1}$ is the orthogonal projection of the origin on $L$, thus

$$
\left\{z_{1}\right\}=L \cap L^{\perp} .
$$

Now let $E_{0}$ be a fixed level and $L$ an affine subspace of the level set $E(x)$ $=E_{0}$,

$$
L=H_{1} \cap\left\{E(x)=E_{0}\right\}, \quad\left\{z_{1}\right\}=\Sigma_{H_{1}} \cap\left\{E(x)=E_{0}\right\} .
$$


Note that since $v_{1} \perp H \cap\left\{E(x)=E_{0}\right\}$, we have

$$
L^{\perp}=H_{1}^{\perp}+\operatorname{Lin}\left(v_{1}\right)=\Pi .
$$

Therefore

$$
\left(H_{1} \cap \Pi\right) \cap\left\{E(x)=E_{0}\right\}=L \cap L^{\perp}=\left\{z_{1}\right\}=\Sigma_{H_{1}} \cap\left\{E(x)=E_{0}\right\} .
$$

This finishes the proof of the corollary.

Note that if $E$ is constant on $H_{1}$ then $\Sigma_{H_{1}}$ consists of one point, otherwise it is a line. Indeed, in the first case $v_{1} \in H_{1}^{\perp}$, hence

$$
\operatorname{dim} \Pi=\operatorname{codim} H_{1},
$$

while in the second case $v_{1} \notin H_{1}^{\perp}$, hence

$$
\operatorname{dim} \Pi=\operatorname{codim} H_{1}+1 .
$$

Note that in both cases the function $X_{H_{1}}$ is unique. In the second case it is a linear function defined on the whole real line $\mathbb{R}$.

Next we shall deal with Markovitz like models. Let $\Delta$ denote a simplex contained in $H, m$ the minimal and $M$ the maximal value of $E$ restricted to $\Delta$. We say that a simplex $\Delta_{1}$ is a subsimplex of $\Delta$ if every vertex of $\Delta_{1}$ is a vertex of $\Delta$. We recall that the matrix $C$ is positive definite and it is well known that the set $\Sigma_{\Delta}$ of portfolios of relatively minimal variance is a polygonal line.

THEOREM 3.2. The set $\Sigma_{\Delta}$ of portfolios of relatively minimal variance is the image of a continuous, piecewise linear (affine) function

$$
X_{\Delta}:[m, M] \rightarrow \Sigma_{\Delta}, \quad E\left(X_{\Delta}(t)\right)=t .
$$

Moreover for any subsimplex $\Delta_{1}$ of $\Delta$ the preimage $X_{\Delta}^{-1}\left(\operatorname{Int} \Delta_{1}\right)$ is connected and the restriction of $X_{\Delta}$ to it coincides with $X_{H_{1}}$ where $H_{1}$ is the affine space spanned by the vertices of $\Delta_{1}$.

\section{Proof}

STEP 1. For every $E \in[m, M]$ there exists exactly one $x_{E} \in \Delta$ such that $E\left(x_{E}\right)=E$ and $V\left(x_{E}\right)=\min \{V(x): x \in \Delta, E(x)=E\}$.

Indeed, since the simplex $\Delta$ is compact and convex, the intersection $\Delta_{E}=\Delta \cap\{E(x)=E\}$ is nonempty, convex and compact. On the compact set $\Delta_{E}$ the continuous function $V$ attains its minimum, $V_{\min }$. Since $C$ is positive definite, it follows that $V$ is strictly convex and $V_{\min }$ is attained at exactly one point.

SteP 2. The mapping $E \mapsto X(E)=X_{\Delta}(E)=x_{E}$ is continuous.

Assume that $X(\cdot)$ is not continuous at some point $E_{0} \in[m, M]$. This means that there exists a sequence $\left(E_{n}\right)_{n=1}^{\infty}$ such that $E_{n} \rightarrow E_{0}$ but $X\left(E_{n}\right)$ $\nrightarrow X\left(E_{0}\right)$. Since $\Delta$ is compact we may choose $E_{n}$ in such a way that 
- it is strictly monotonic,

- $X\left(E_{n}\right)$ has a limit

$$
\lim _{n \rightarrow \infty} X\left(E_{n}\right)=x_{\infty}
$$

Obviously $E\left(x_{\infty}\right)=E_{0}$. Therefore since $X\left(E_{0}\right)$ is a unique minimum of $V$ on $\Delta_{E_{0}}$ the only possibility is that $V\left(x_{\infty}\right)>V\left(X\left(E_{0}\right)\right)$. To show that this is not possible we consider the segment of portfolios

$$
x_{t}=t X\left(E_{1}\right)+(1-t) X\left(E_{0}\right), \quad t \in[0,1] .
$$

Since $V$ is convex,

$$
V\left(x_{t}\right) \leq t V\left(X\left(E_{1}\right)\right)+(1-t) V\left(X\left(E_{0}\right)\right) .
$$

Since our sequence $E_{n}$ is strictly monotonic we get for every $n>1$ the estimate

$$
V\left(X\left(E_{n}\right)\right) \leq V\left(x_{t_{n}}\right)<t_{n} V\left(X\left(E_{1}\right)\right)+\left(1-t_{n}\right) V\left(X\left(E_{0}\right)\right),
$$

where $t_{n} \in(0,1)$ is a solution of the equation

$$
E_{n}=t_{n} E_{1}+\left(1-t_{n}\right) E_{0} .
$$

Letting $n$ tend to infinity we get

$$
V\left(x_{\infty}\right) \leq V\left(X\left(E_{0}\right)\right)
$$

which eliminates the above possibility. We get a contradiction.

Step 3. $X_{\Delta}(\cdot)$ is piecewise linear.

If $E(\cdot)$ is constant on $H$ then $\Sigma_{\Delta}$ consists of one point. Otherwise we apply induction on the number of vertices of $\Delta$.

Assume that for every proper subsimplex $\Delta^{\prime}$ of $\Delta$ the set of portfolios of relatively minimal variance is a union of segments or a point. Since $E(\cdot)$ is not constant on $H$, from Corollary 3.1 we see that the intersection of $\Sigma_{\Delta}$ and the interior of $\Delta$ is either empty or an open segment. The rest of $\Sigma_{\Delta}$ is contained in the border of $\Delta$, i.e. the union of its proper subsimplexes. Since $\Sigma_{\Delta} \cap \Delta^{\prime} \subset \Sigma_{\Delta^{\prime}}$ we get

$$
\Sigma_{\Delta} \subset\left(\Sigma_{\Delta} \cap \operatorname{Int}(\Delta)\right) \cup \bigcup \Sigma_{\Delta^{\prime}}
$$

Hence $\Sigma_{\Delta}$ is contained in a finite union of segments and points. Since it is the image of a continuous function $X_{\Delta}(\cdot)$, this function must be piecewise linear.

STEP 4. For any subsimplex $\Delta_{1}$ of $\Delta$ the restriction of $X_{\Delta_{1}}$ to the preimage $X_{\Delta_{1}}^{-1}\left(\operatorname{Int} \Delta_{1}\right)$ coincides with the restriction of $X_{H_{1}}$ where $H_{1}$ is the affine space spanned by the vertices of $\Delta_{1}$.

It is enough to show that the images of both functions coincide. One inclusion is obvious:

$$
\Delta_{1} \subset H_{1} \Rightarrow \Sigma_{H_{1}} \cap \Delta_{1} \subset \Sigma_{\Delta_{1}} \Rightarrow \Sigma_{H_{1}} \cap \operatorname{Int} \Delta_{1} \subset \Sigma_{\Delta_{1}} \cap \operatorname{Int} \Delta_{1} .
$$


The other inclusion follows from the fact that Int $\Delta_{1}$ is an open subset of $H_{1}$. Indeed, assume that there exists $t$ such that

$$
X_{\Delta_{1}}(t) \in \operatorname{Int} \Delta_{1}
$$

Now, Int $\Delta_{1} \cap\{E(x)=t\}$ is an open subset of the affine space $H_{1} \cap\{E(x)$ $=t\}$. Since the function $V$ restricted to Int $\Delta_{1} \cap\{E(x)=t\}$ has a global minimum at the point $X_{\Delta_{1}}(t)$, the function $V$ restricted to $H_{1} \cap\{E(x)=t\}$ has at least a local minimum at this point. But $V$ is strictly convex and a local minimum must be a global one, i.e.

$$
X_{H_{1}}(t)=X_{\Delta_{1}}(t) \text {. }
$$

STEP 5. For any two subsimplexes $\Delta_{1}$ and $\Delta_{2}$ of $\Delta$ with $\Delta_{1} \subset \Delta_{2} \subset \Delta$, the intersection $\Sigma_{\Delta_{2}} \cap$ Int $\Delta_{1}$ is connected.

Assume that the above statement is not true, and select the smallest subsimplex $\Delta_{3}$ of $\Delta$ such that $\Delta_{1} \subset \Delta_{3}$ and $\Sigma_{\Delta_{3}} \cap \operatorname{Int} \Delta_{1}$ is not connected. Due to the minimality of $\Delta_{3}$ there are real numbers $E_{1}<E_{2}$ such that

$$
X_{\Delta_{3}}\left(E_{1}\right) \in \operatorname{Int} \Delta_{1}, \quad X_{\Delta_{3}}\left(E_{2}\right) \in \operatorname{Int} \Delta_{1}, \quad \forall t \in\left(E_{1}, E_{2}\right) \quad X_{\Delta_{3}}(t) \in \operatorname{Int} \Delta_{3} .
$$

Let $H_{i}$ be the affine space spanned by the vertices of $\Delta_{i}$. Since the functions $X_{*}$ are continuous we get

$$
X_{H_{3}}\left(E_{j}\right)=X_{\Delta_{3}}\left(E_{j}\right)=X_{\Delta_{1}}\left(E_{j}\right)=X_{H_{1}}\left(E_{j}\right), \quad j=1,2 .
$$

But two linear functions $X_{H_{*}}$ equal at two different points must be equal, which leads to a contradiction.

REMARK. Since the critical set $\Sigma_{\Delta}$ of portfolios of relatively minimal variance is the image of a piecewise linear mapping it is called the polygonal line of portfolios of relatively minimal variance.

EXAMPLE 3.1 ("Noncorrelated rates of return"). We consider the simplest $n$-dimensional model describing the assets with noncorrelated (for example independent) rates of return. Here $C=\mathrm{Id}, \Delta=\left\{x: \sum x_{i}=1\right.$, $\left.x_{i} \geq 0\right\}=\Delta\left(e_{1}, \ldots, e_{n}\right)$, where $e_{i}$ are the unit vectors, $h=e=(1, \ldots, 1)^{T}$ and $H=\left\{x: e^{T} x=1\right\}$. We assume that the expected rates of return of different assets are different, and we order the assets according to these rates:

$$
\mu=\left(\mu_{1}, \ldots, \mu_{n}\right)^{T}, \quad \mu_{1}<\ldots<\mu_{n} .
$$

In this case the basic quantities can be expressed in terms of the mean $\bar{\mu}$ and the standard deviation $S_{\mu}$ of $\mu_{i}$ 's:

$$
\begin{gathered}
\alpha=\sum \mu_{i}^{2}, \quad \beta=\sum \mu_{i}=n \bar{\mu}, \quad \gamma=n, \\
\delta=n \sum \mu_{i}^{2}-\left(\sum \mu_{i}\right)^{2}=n^{2} S_{\mu}^{2}, \\
v_{0}=\frac{1}{\sqrt{n}} e, \quad v_{1}=\frac{1}{\sqrt{n} S_{\mu}}(\mu-\bar{\mu} e),
\end{gathered}
$$




$$
X_{H}(t)=\frac{1}{\sqrt{n}} v_{0}+(t-\bar{\mu}) \frac{1}{\sqrt{n} S_{\mu}} v_{1}=\frac{1}{n}\left(e+\frac{t-\bar{\mu}}{S_{\mu}^{2}}(\mu-\bar{\mu} e)\right), \quad t \in \mathbb{R} .
$$

We show that:

1. The critical line $\Sigma$ intersects the interior of $\Delta$.

2. The points

$$
\begin{aligned}
& q_{1}=X_{H}\left(t_{1}\right), \quad t_{1}=\bar{\mu}-\frac{S_{\mu}^{2}}{\mu_{n}-\bar{\mu}}, \\
& q_{2}=X_{H}\left(t_{2}\right), \quad t_{2}=\bar{\mu}-\frac{S_{\mu}^{2}}{\mu_{1}-\bar{\mu}},
\end{aligned}
$$

are the points of intersection of $\Sigma$ and the boundary of $\Delta$.

3. $q_{1}$ and $q_{2}$ belong to subsimplexes of codimension 1 ,

$$
q_{2} \in \Delta_{1}=\Delta\left(e_{2}, \ldots, e_{n}\right), \quad q_{1} \in \Delta_{n}=\Delta\left(e_{1}, \ldots, e_{n-1}\right) .
$$

4. The parametrization of the polygonal line $\Sigma_{\Delta}$ decomposes as follows:

$$
X_{\Delta}(t)= \begin{cases}X_{\Delta_{n}}(t) & \text { for } t \in\left[\mu_{1}, t_{1}\right] \\ X_{H}(t) & \text { for } t \in\left(t_{1}, t_{2}\right) \\ X_{\Delta_{1}}(t) & \text { for } t \in\left[t_{2}, \mu_{n}\right]\end{cases}
$$

To do this we have to check for which $t$ the coordinates of the parametrization $X_{H}(t)$ are positive. For $j=1, \ldots, n$ the $j$ th coordinate equals

$$
e_{j}^{T} X_{H}(t)=\frac{1}{n}\left(1+(t-\bar{\mu}) \frac{\mu_{j}-\bar{\mu}}{S_{\mu}^{2}}\right) \geq 0 .
$$

The condition imposed on $t$ depends on the sign of $\mu_{j}-\bar{\mu}$. We obtain:

- for $\mu_{j}<\bar{\mu}$,

$$
t \leq \bar{\mu}-\frac{S_{\mu}^{2}}{\mu_{j}-\bar{\mu}}
$$

- for $\mu_{j}=\bar{\mu}$ there is no restriction on $t$;

- for $\mu_{j}>\bar{\mu}$,

$$
t \geq \bar{\mu}-\frac{S_{\mu}^{2}}{\mu_{j}-\bar{\mu}}
$$

Taking the smallest and largest $\mu_{j}$, i.e. $\mu_{1}$ and $\mu_{n}$, we get the formulas for $t_{1}$ and $t_{2}$ :

$$
t_{1}=\bar{\mu}-\frac{S_{\mu}^{2}}{\mu_{n}-\bar{\mu}}, \quad t_{2}=\bar{\mu}-\frac{S_{\mu}^{2}}{\mu_{1}-\bar{\mu}} .
$$

For $t \in\left[t_{1}, t_{2}\right], X_{H}(t)$ belongs to the simplex $\Delta$. Moreover only the last coordinate of $X_{H}\left(t_{1}\right)$ and the first of $X_{H}\left(t_{2}\right)$ vanish. Hence $X_{H}\left(t_{1}\right)$ belongs to the face $\Delta\left(e_{1}, \ldots, e_{n-1}\right)$; and $X_{H}\left(t_{2}\right)$ belongs to $\Delta\left(e_{2}, \ldots, e_{n}\right)$.

The fourth property follows from the fact that the first and last coordinates of $X_{\Delta}(t)$ are monotonic. Indeed, $X_{\Delta}(\cdot)$ is a continuous piecewise 
linear function. Moreover each linear part is equal to $X_{H^{\prime}}$ where $H^{\prime}$ is an affine space containing the subsimplex $\Delta^{\prime}$ of $\Delta$. There are two possibilities. If $e_{1}$ (resp. $\left.e_{n}\right)$ is not a vertex of $\Delta^{\prime}$ then the corresponding coordinate is constant, equal to 0 . Otherwise, since the restriction of the noncorrelated returns model to a subsimplex remains of the same type, we get the monotonicity from the above formula for $e_{j}^{T} X_{H}(t)$.

4. Generic models. The above theorems on normal forms suggest the following notion of "general position".

The linear function $E$ is in general position if it is not constant on $\Delta$. The quadratic function $V$ is in general position if the critical line $\Sigma$ is in general position with respect to the skeleton of $\Delta$. Adding to the above the hereditary condition we obtain:

Definition. The $n$-dimensional mean-variance model $(\Delta, E, V)$ is generic if

- $\Delta$ is an $(n-1)$-dimensional simplex,

- the values of $E$ at the vertices of $\Delta$ are pairwise different,

- for any two affine spaces $H_{1} \subset H_{2}$ spanned by some vertices of $\Delta$, if $2+\operatorname{dim} H_{1} \leq \operatorname{dim} H_{2}$ then the critical line $\Sigma_{H_{2}}$ neither intersects $H_{1}$ nor is parallel to it.

REMARK. 1. The restriction of a generic model to a face of the simplex $\Delta$ remains generic.

2. In the generic case the mean $E$ is not constant on any subsimplex of $\Delta$ (of positive dimension).

3. All one-dimensional models are generic.

4. A two-dimensional model is generic if and only if $E\left(z_{1}\right) \neq E\left(z_{2}\right)$, where $z_{i}$ are vertices of $\Delta$.

Note that if $\Delta$ has less than 3 vertices then the affine space spanned by some vertices of $\Delta$ has dimension smaller than 2 and the third condition is meaningless.

The mean-variance model $(\Delta, E, V)$ is determined by the vertices of $\Delta$, a vector $\mu$ and a positive definite, symmetric matrix $C$. Therefore the set $\mathcal{M}$ of all $n$-dimensional mean-variance models, with $\Delta$ being an $(n-1)$ dimensional simplex, is parametrized by an open semialgebraic subset of $\mathbb{R}^{N}$, where

$$
N=n^{2}+n+\frac{n(n+1)}{2}=\frac{3 n(n+1)}{2} .
$$

Having the topology on $\mathcal{M}$ (induced by the parametrization) we may introduce equivalence of generic models basing on continuous deformations. Namely: 
Definition. Two generic $n$-dimensional models $(\Delta, E, V),\left(\Delta^{\prime}, E^{\prime}, V^{\prime}\right)$ are equivalent if there exists a continuous path

$$
\phi:[0,1] \rightarrow \mathbb{R}^{N}, \quad t \mapsto\left(z_{1, t}, \ldots, z_{n, t}, \mu_{t}, C_{t}\right),
$$

such that:

$$
\begin{aligned}
& \Delta=\Delta\left(z_{1,0}, \ldots, z_{n, 0}\right), \quad E(x)=\mu_{0}^{T} x, \quad V(x)=x^{T} C_{0} x \quad \text { for } x \in \mathbb{R}^{n}, \\
& \Delta^{\prime}=\Delta\left(z_{1,1}, \ldots, z_{n, 1}\right), \quad E^{\prime}(x)=\mu_{1}^{T} x, \quad V^{\prime}(x)=x^{T} C_{1} x \quad \text { for } x \in \mathbb{R}^{n},
\end{aligned}
$$

and for all $t \in[0,1],\left(\Delta\left(z_{1, t}, \ldots, z_{n, t}\right), \mu_{t}^{T} x, x^{T} C_{t} x\right)$ is a generic model.

In the next sections we will show that the set $\mathcal{M}_{\mathrm{g}}$ of generic models is also an open semialgebraic subset of $\mathbb{R}^{N}$, and moreover it is dense in $\mathcal{M}$. Therefore, as a matter of fact, two generic models are equivalent if and only if they belong to the same connected component of $\mathcal{M}_{\mathrm{g}}$.

REMARK. The equivalence relation introduced above can be extended to nongeneric cases. Namely we stratify the space of all models depending on the violated regularity conditions and say that two models are equivalent if one can be deformed to the other within the same stratum.

5. Lines of relative minimal variance for generic models. The aim of this section is to show that equivalent generic models have similar polygonal lines of portfolios of relatively minimal variance, namely these lines cross the same subsimplexes. For simplicity we apply the coordinate system determined by the vertices of the simplex $\Delta$. Throughout this section the $i$ th vertex of $\Delta$ is the unit vector of the $i$ th axis, i.e. $\Delta=\Delta\left(e_{1}, \ldots, e_{n}\right)$, where $e_{i}$ is a vector with one nonzero coefficient, equal to 1 , at the $i$ th position. Furthermore we code subsimplexes by their characteristic functions. The $\{0,1\}$ sequence $\left(\varepsilon_{1}, \ldots, \varepsilon_{n}\right)$ denotes the simplex spanned by the vertices $e_{i}$ with $\varepsilon_{i}=1$.

We shall investigate three functions induced by $X_{\Delta}$ :

$$
\begin{aligned}
\operatorname{sim}:[m, M] & \rightarrow\{0,1\}^{n}, \\
\operatorname{dim}:[m, M] & \rightarrow\{0,1, \ldots, n-1\}, \\
l_{2}:[m, M] & \rightarrow(0,1] .
\end{aligned}
$$

Let $t \in[m, M]$. Then $\operatorname{sim}(t)$ is the subsimplex whose interior contains $X_{\Delta}(t), \operatorname{dim}(t)$ is its dimension, and $l_{2}(t)$ the second smallest positive coordinate of $X_{\Delta}(t)$ if it is not a vertex and 1 otherwise.

The functions sim, dim and $l_{2}$ are compositions of $X_{\Delta}$ and functions defined on the unit cube:

$$
\operatorname{sim}(t)=\operatorname{Sgn} \circ X_{\Delta}(t), \quad \operatorname{dim}(t)=\Sigma \operatorname{sgn} \circ X_{\Delta}(t), \quad l_{2}(t)=L_{2} \circ X_{\Delta}(t),
$$


where

$$
\begin{aligned}
\operatorname{Sgn}\left(x_{1}, \ldots, x_{n}\right) & =\left(\operatorname{sgn}\left(x_{1}\right), \ldots, \operatorname{sgn}\left(x_{n}\right)\right), \\
\Sigma \operatorname{sgn}\left(x_{1}, \ldots, x_{n}\right) & =\operatorname{sgn}\left(x_{1}\right)+\ldots+\operatorname{sgn}\left(x_{n}\right), \\
L_{2}\left(x_{1}, \ldots, x_{n}\right) & = \begin{cases}\min \left\{l: \#\left\{i: 0<x_{i} \leq l\right\}=2\right\} & \text { if } \#\left\{i: x_{i}>0\right\}>1, \\
1 & \text { otherwise. }\end{cases}
\end{aligned}
$$

The function $\operatorname{dim}(t)$ is lower semicontinuous (because $\Sigma \operatorname{sgn}\left(x_{1}, \ldots, x_{n}\right.$ ) is lower semicontinuous for nonnegative $x_{i}$ 's). Moreover for a generic model the jumps of $\operatorname{dim}(t)$ are not greater than 1 .

LEMMA 5.1. For every generic model the function dim is either locally constant or has jumps of size 1 , i.e.

$$
\begin{gathered}
\forall t_{0} \in[m, M] \exists \varepsilon>0 \forall t \in[m, M] \cap\left(t_{0}-\varepsilon, t_{0}+\varepsilon\right) \\
\operatorname{dim}(t)=\operatorname{dim}\left(t_{0}\right) \quad \text { or } \quad \operatorname{dim}(t)=\operatorname{dim}\left(t_{0}\right)+1 .
\end{gathered}
$$

Proof. Let $\Delta_{1}$ be a subsimplex of $\Delta$ such that $X_{\Delta}\left(t_{0}\right)$ belongs to Int $\Delta_{1}$. Then either the intersection $\Sigma_{\Delta} \cap \operatorname{Int} \Delta_{1}$ is a segment (not one point!) and $X_{\Delta}\left(t_{0}\right)$ is its inner point, or $X_{\Delta}\left(t_{0}\right)$ is approached by $X_{\Delta}(t)$ from the interior of some larger subsimplex, say $\Delta_{2}$. In the first case $\operatorname{dim}(t)$ is constant on some neighbourhood of $t_{0}$ (Theorem 3.2). In the second case there is a jump. But since $X_{\Delta}$ is continuous, we have

$$
X_{\Delta}\left(t_{0}\right) \in \operatorname{cl}\left(\Sigma_{\Delta} \cap \operatorname{Int} \Delta_{2}\right) \subset \operatorname{cl}\left(\sigma_{H_{2}} \cap \operatorname{Int} \Delta_{2}\right) \subset \Sigma_{H_{2}},
$$

where cl stands for closure, and $H_{2}$ is the affine space spanned by $\Delta_{2}$. Due to the generic conditions $\Sigma_{H_{2}}$ cannot intersect subsimplexes of dimension smaller than $\operatorname{dim} H_{2}-1$. Therefore the jump is equal to 1.

EXAMPLE 5.1. We describe the $\operatorname{sim}(t)$ function for the "noncorrelated rates of return" model from Example 3.1. The polygonal line of portfolios of relatively minimal variance starts from the vertex $e_{1}$, crosses $2 n-1$ open subsimplexes and ends at $e_{n}$ :

$$
\operatorname{sim}(t)=\left\{\begin{array}{cl}
(1,0,0, \ldots, 0,0,0) & \text { if } t=t_{1} \\
(1,1,0, \ldots, 0,0,0) & \text { if } t \in\left(t_{1}, t_{2}\right] \\
\ldots & \\
(1,1,1, \ldots, 1,1,0) & \text { if } t \in\left(t_{n-2}, t_{n-1}\right] \\
(1,1,1, \ldots, 1,1,1) & \text { if } t \in\left(t_{n-1}, t_{n}\right), \\
(0,1,1, \ldots, 1,1,1) & \text { if } t \in\left[t_{n}, t_{n+1}\right), \\
(0,0,0, \ldots, 0,1,1) & \text { if } t \in\left[t_{2 n-3}, t_{2 n-2}\right), \\
(0,0,0, \ldots, 0,0,1) & \text { if } t=t_{2 n-2},
\end{array}\right.
$$


where $t_{1}=\mu_{1}, t_{2 n-2}=\mu_{n}$, and for $j=3, \ldots, n$,

$$
t_{j-1}=\bar{\mu}_{j}-\frac{S_{j}}{\mu_{j}-\bar{\mu}_{j}}, \quad t_{2 n-j}=\bar{\mu}_{j}^{*}-\frac{S_{j}^{*}}{\mu_{n-j+1}-\bar{\mu}_{j}^{*}} .
$$

Here $\bar{\mu}_{j}$ and $S_{j}$ are the mean and standard deviation of the first $j \mu_{i}$ 's, while $\bar{\mu}_{j}^{*}$ and $S_{j}^{*}$ are the mean and standard deviation of the last $j \mu_{i}$ 's.

Indeed, as was shown in Example 3.1, $\Sigma_{\Delta}$ intersects the interior of the simplex $\Delta=(1, \ldots, 1)$. Moreover starting from this interior one can get along $\Sigma_{\Delta}$ only to the open simplexes $(0,1, \ldots, 1)$ or $(1, \ldots, 1,0)$. Next we continue by induction.

The values of $t_{i}$ are obtained from Example 3.1 applied to the subsimplexes $(1, \ldots, 1,0, \ldots, 0)$ and $(0, \ldots, 0,1, \ldots, 1)$.

Lemma 5.2. Every generic model $(\Delta, E, V)$ has a neighbourhoodU in the space of all generic models on which the function $l_{2}$ is uniformly bounded away from 0 :

$$
\exists \varepsilon>0 \forall\left(\Delta^{\prime}, E^{\prime}, V^{\prime}\right) \in \mathcal{U} \forall t \quad l_{2}(t) \geq \varepsilon
$$

Proof. Since $\Delta$ is covered by a finite number of open subsimplexes, it is enough to find a positive lower bound $\varepsilon$ for every subsimplex $\Delta_{1}$. Let $H_{1}$ be the affine space spanned by the vertices of $\Delta_{1}$. We have the inclusion (see Theorem 3.2)

$$
\Sigma_{\Delta} \cap \operatorname{Int} \Delta_{1} \subset \Sigma_{H_{1}} \cap \operatorname{Int} \Delta_{1} .
$$

Assume that $\Sigma_{H_{1}}$ crosses the interior of $\Delta_{1}$ (otherwise we put $\varepsilon=1$ ). Due to the generic conditions the critical line $\Sigma_{H_{1}}$ crosses only the faces of $\Delta_{1}$ of codimension 1 . Hence at a crossing point only one coordinate tends to 0 . Therefore $L_{2}$ is bounded away from 0 on $\Sigma_{H_{1}} \cap \operatorname{Int} \Delta_{1}$. Moreover $\Sigma_{H_{1}}$ depends continuously on the coefficients of the model. Thus we can find a uniform bound.

The final estimate is the minimum of all bounds obtained for subsimplexes.

Lemma 5.3. Let $(\Delta, E, V)$ be a generic model. If a subsimplex $\Delta_{1}$ of $\Delta$ has at least two vertices and the intersection $\Pi=\Sigma_{\Delta} \cap \operatorname{Int} \Delta_{1}$ is not empty then $\Pi$ is a segment.

Proof. Since $\Pi=\Sigma_{\Delta} \cap \operatorname{Int} \Delta_{1}$ is a bounded, connected subset of a line (see Theorem 3.2) it can be either an empty set, a point or a segment. We show that in the case of a generic model it cannot be a point. Assume the contrary:

$$
\Sigma_{\Delta} \cap \operatorname{Int} \Delta_{1}=\{q\} .
$$

Then there are two bigger simplexes, say $\Delta_{2}$ and $\Delta_{3}$, such that for $t$ close to $E(q), X_{\Delta}(t)$ belongs to one of their interiors. Let $\Delta_{4}$ be the simplex spanned 
by the vertices of both $\Delta_{2}$ and $\Delta_{3}$, and $H_{i}$ the affine space spanned by the vertices of $\Delta_{i}, i=1,2,3,4$. Obviously

$$
H_{1}=H_{2} \cap H_{3}, \quad H_{4} \supset H_{2} \cup H_{3} .
$$

Furthermore, let $\Pi_{i}=\operatorname{Lin}\left(v_{1}\right)+H_{i}^{\perp}$. As shown in Corollary 3.2,

$$
\Sigma_{H_{i}}=H_{i} \cap \Pi_{i} \text {. }
$$

Moreover, $q \in \Pi_{2} \cap \Pi_{3}$.

We estimate the dimension of $\Pi_{1}$. We have

$\Pi_{1}=\operatorname{Lin}\left(v_{1}\right)+H_{1}^{\perp}=\operatorname{Lin}\left(v_{1}\right)+\left(H_{2} \cap H_{3}\right)^{\perp}=\operatorname{Lin}\left(v_{1}\right)+H_{2}^{\perp}+H_{1}^{\perp}=\Pi_{2}+\Pi_{3}$.

Hence

$$
\operatorname{dim} \Pi_{1}=\operatorname{dim} \Pi_{2}+\operatorname{dim} \Pi_{3}-\operatorname{dim} \Pi_{2} \cap \Pi_{3} .
$$

But $\Pi_{2} \cap \Pi_{3} \supset \Pi_{4} \cup\{q\}$. Since our model is generic, $q \notin \Pi_{4}$. Indeed, $q \in H_{1} \subset H_{4}$ and $\Pi_{4} \cap H_{4}=\Sigma_{H_{4}}$, which does not intersect $H_{1}\left(\operatorname{dim} H_{1} \leq\right.$ $\left.\operatorname{dim} H_{4}-2\right)$. Therefore

$$
\operatorname{dim} \Pi_{2} \cap \Pi_{3} \geq \operatorname{dim} \Pi_{4}+1 .
$$

Hence

$$
\operatorname{dim} \Pi_{1} \leq \operatorname{codim} H_{2}+1+\operatorname{codim} H_{3}+1-\left(\operatorname{codim} H_{4}+2\right)=\operatorname{codim} H_{1} .
$$

From Theorem 3.1 it follows that $E$ is constant on $H_{1}$, which is not possible because the model is generic.

We recall that two functions $\phi_{i}:\left[m_{i}, M_{i}\right] \rightarrow \mathbb{R}, i=1,2$, are right piecewise linear equivalent if there exists a piecewise linear homeomorphism

$$
\psi:\left[m_{1}, M_{1}\right] \stackrel{\text { onto }}{\longrightarrow}\left[m_{2}, M_{2}\right], \quad \psi\left(m_{1}\right)=m_{2},
$$

such that

$$
\phi_{2} \circ \psi=\phi_{1} .
$$

TheOREM 5.1. For equivalent generic models the corresponding functions sim are right piecewise linear equivalent.

Proof. Since there is a continuous deformation $\left(\Delta, E_{s}, V_{s}\right), s \in[0,1]$, from one model to the other, there is also a continuous family of $X_{\Delta}$ functions

$$
X_{s}:\left[m_{s}, M_{s}\right] \rightarrow \Delta, \quad s \in[0,1] .
$$

We deform $X_{s}$ into the skeleton of the unit cube. Let $H_{r}: \Delta \rightarrow[0,1]^{n}$ denote the deformation

$$
\begin{aligned}
H_{r}\left(x_{1}, \ldots, x_{n}\right)=\left(\min \left(1, r x_{1}\right), \ldots,\right. & \left.\min \left(1, r x_{n}\right)\right), \\
r & \quad \in[1, \infty), x=\left(x_{1}, \ldots, x_{n}\right) \in \Delta .
\end{aligned}
$$

Note that $H_{r}$ "preserves" $\operatorname{sim}_{s}$, namely for every $r \geq 1$ and $t \in\left[m_{s}, M_{s}\right]$,

$$
\operatorname{sim}_{s}(t)=\operatorname{Sgn}\left(H_{r}\left(X_{s}(t)\right)\right) \text {. }
$$


Since the closed interval is compact and all models are generic there is a uniform bound $B$ for the second smallest coordinate of $\Sigma_{s}$. Obviously for every $s$ and $r>1 / B$ the image of $H_{r} X_{s}$ is contained in the one-dimensional skeleton of the cube. Since $X_{s}$ is piecewise linear and it crosses the interior of any subsimplex at most once, for $r>1 / B$ the mappings $H_{r} X_{s}$ are right piecewise linear equivalent.

Now $\operatorname{sim}_{s}=\operatorname{Sgn} \circ H_{r} \circ X_{s}$, therefore $\operatorname{sim}_{s}$ 's are right piecewise linearly equivalent.

6. Invariants. The aim of this section is to provide certain numerical invariants of the equivalence relation of generic models, namely the signs of the $n$-minors of certain $n \times(2 n+2)$ matrices of the form $(a, b, A, B)$ where $A$ and $B$ are invertible square $n \times n$-matrices and $a, b$ are column vectors.

Let $\mathcal{I}$ and $\mathcal{J}$ be two sets of indices, $\mathcal{I}, \mathcal{J} \subset\{1, \ldots, n\}$, having respectively $k$ and $n-k-2$ elements, $k=0,1, \ldots, n-2$. We denote by $d_{\mathcal{I}, \mathcal{J}}$ the $n$-minor of the $n \times(2 n+2)$ matrix $(a, b, A, B)$ obtained by taking: first the two columns $a$ and $b$, then the $\mathcal{I}$ columns of $A$ and the $\mathcal{J}$ columns of $B$ (preserving the order of the columns).

Definition. We call the minors $d_{\mathcal{I}, \mathcal{J}}$ admissible if the sets $\mathcal{I}$ and $\mathcal{J}$ are disjoint.

With every $n$-dimensional mean-variance model $(\Delta, E, V)$ such that $\Delta$ is a simplex spanned by $n$ linearly independent vertices $z_{1}, \ldots, z_{n}$, we associate four $n \times(2 n+2)$ matrices $C^{\#}, Z^{\#}, Z_{1}^{\#}$ and $Y^{\#}$ as follows:

- $C^{\#}=(e, \widehat{\mu}, \widehat{C}, \mathrm{Id})$, where $e=(1, \ldots, 1)^{T}, \widehat{\mu}=\left(E\left(z_{1}\right), \ldots E\left(z_{n}\right)\right)^{T}$, and $\widehat{C}$ is the Gram matrix of the vertices of $\Delta, \widehat{C}_{i, j}=z_{i}^{T} C z_{j}$.

- $Z^{\#}=\left(h, \mu, C Z,\left(Z^{-1}\right)^{T}\right)$, where $Z$ is the matrix of the coefficients of the vertices $z_{i}$ of the simplex $\Delta, Z=\left(z_{1}, \ldots, z_{n}\right)$. Note that $Z^{T} h=e$, $Z^{T} \mu=\widehat{\mu}$ and $Z^{T} C Z=\widehat{C}$.

- $Z_{1}^{\#}=\left(v_{0}, v_{1}, Z, Z^{*}\right)$, where $Z^{*}$ is the matrix of the coefficients of the dual basis, $Z^{*}=\left(z_{1}^{*}, \ldots, z_{n}^{*}\right)$. The duals $z_{i}^{*}$ are determined by the rule

$$
z_{j}^{T} C z_{i}^{*}= \begin{cases}0 & \text { if } i \neq j \\ 1 & \text { if } i=j .\end{cases}
$$

Note that since $Z^{T} C Z^{*}=\mathrm{Id}$, we have $Z^{*}=C^{-1}\left(Z^{-1}\right)^{T}$.

- $Y^{\#}=\left(e_{1}, e_{2}, Y,\left(Y^{-1}\right)^{T}\right)$, where $Y$ is the matrix of the $y$-coordinates of the vertices $z_{i}$ (see $\S 3$ ) and $e_{i}$ is the $i$ th standard unit vector.

- $Y=\Phi Z$, where $\Phi$ is a transition matrix. Since $Z^{T} C Z=\widehat{C}=Y^{T} Y$, we have $C=\Phi^{T} \Phi$. 
We recall that $\Phi v_{0}=e_{1}$ and $\Phi v_{1}=e_{2}$. Therefore

$$
\begin{aligned}
& Y^{T} e_{1}=Y^{T} \Phi v_{0}=Z^{T} \Phi^{T} \Phi v_{0}=Z^{T} C v_{0}=\frac{1}{\sqrt{\gamma}} e \\
& Y^{T} e_{2}=Y^{T} \Phi v_{1}=Z^{T} \Phi^{T} \Phi v_{1}=Z^{T} C v_{1}=\frac{1}{\sqrt{\gamma \delta}}(\gamma \widehat{\mu}-\beta e) .
\end{aligned}
$$

We show that the signs of the $d_{\mathcal{I}, \mathcal{J}}$ minors of the above matrices are closely related.

LEMMA 6.1. For any disjoint $\mathcal{I}, \mathcal{J}$,

$$
\begin{aligned}
d_{\mathcal{I}, \mathcal{J}}\left(C^{\#}\right) & =\sqrt{\delta} \operatorname{det} Y \cdot d_{\mathcal{I}, \mathcal{J}}\left(Y^{\#}\right)=\operatorname{det} Z \cdot d_{\mathcal{I}, \mathcal{J}}\left(Z^{\#}\right) \\
& =\sqrt{\delta} \operatorname{det} Z \cdot \operatorname{det} C \cdot d_{\mathcal{I}, \mathcal{J}}\left(Z_{1}^{\#}\right) .
\end{aligned}
$$

Proof. Multiplication of an $n \times n$ square matrix by an $n \times(n+2)$ matrix induces multiplication of $n$-minors of the second matrix by the determinant of the first. Hence the assertion of the lemma follows from the equalities

$$
\begin{aligned}
C Z_{1}^{\#} & =C\left(v_{0}, v_{1}, Z, Z^{*}\right)=\left(\frac{1}{\sqrt{\gamma}} h, \frac{1}{\sqrt{\gamma \delta}}(\gamma \mu-\beta h), C Z,\left(Z^{-1}\right)^{T}\right), \\
Z^{T} Z^{\#} & \left.=Z^{T}\left(h, \mu, C Z,\left(Z^{-1}\right)^{T}\right)\right)=(e, \widehat{\mu}, \widehat{C}, \mathrm{Id})=C^{\#}, \\
Y^{T} Y^{\#} & =Y\left(e_{1}, e_{2}, Y,\left(Y^{-1}\right)^{T}\right)=\left(\frac{1}{\sqrt{\gamma}} e, \frac{1}{\sqrt{\gamma \delta}}(\gamma \widehat{\mu}-\beta e), \widehat{C}, \mathrm{Id}\right) .
\end{aligned}
$$

COROLlary 6.1. If $\operatorname{det} Z>0$ and $\operatorname{det} Y>0$ then the corresponding $d_{\mathcal{I}, \mathcal{J}}$ minors of the matrices $C^{\#}, Z^{\#}, Z_{1}^{\#}$ and $Y^{\#}$ have the same sign.

The dual basis simplifies the description of $C$-orthogonal spaces. Let $H\left(z_{i}: i \in \mathcal{I}\right)\left(\operatorname{Lin}\left(v_{0}, z_{j}^{*}: j \notin \mathcal{I}\right), \operatorname{Lin}\left(v_{0}, v_{1}, z_{j}^{*}: j \notin \mathcal{I}\right)\right)$ be the affine (respectively linear) space spanned by the vertices $z_{i}$ where $i \in \mathcal{I}$ (resp. by $v_{0}$ and $z_{j}^{*}$ where $j \notin \mathcal{I}$, or $v_{0}, v_{1}$ and $z_{j}^{*}$ where $j \notin \mathcal{I}$ ). Our proof of the main theorem on invariants will be based on the following:

LEMMA 6.2. For any set of vertices $z_{i}, i \in \mathcal{I}$, of $\Delta$,

$$
H\left(z_{i}: i \in \mathcal{I}\right)^{\perp}=\operatorname{Lin}\left(v_{0}, z_{j}^{*}: j \notin \mathcal{I}\right) .
$$

Proof. We recall that for $i \in \mathcal{I}$ and $j \notin \mathcal{I}, z_{i}^{T} C z_{j}^{*}=0$ and $z_{i}^{T} C v_{0}=1 / \sqrt{\gamma}$. Therefore $v_{0}$ and $z_{j}^{*}$ 's are orthogonal to $H\left(z_{i}: i \in \mathcal{I}\right)$ but $v_{0}$ does not belong to $\operatorname{Lin}\left(z_{j}^{*}: j \notin \mathcal{I}\right)$. We get

$$
H\left(z_{i}: i \in \mathcal{I}\right)^{\perp} \supset \operatorname{Lin}\left(v_{0}, z_{j}^{*}: j \notin \mathcal{I}\right),
$$

but

$$
\begin{aligned}
\operatorname{dim} \operatorname{Lin}\left(v_{0}, z_{j}^{*}: j \notin \mathcal{I}\right) & =n-\# \mathcal{I}+1=\operatorname{codim} H\left(z_{i}: i \in \mathcal{I}\right) \\
& =\operatorname{dim} H\left(z_{i}: i \in \mathcal{I}\right)^{\perp}
\end{aligned}
$$

Therefore the two spaces coincide. 
COROllary 6.2. For any set of vertices $z_{i}, i \in \mathcal{I}$, of $\Delta$,

$$
\Sigma_{H\left(z_{i}: i \in \mathcal{I}\right)}=H\left(z_{i}: i \in \mathcal{I}\right) \cap \operatorname{Lin}\left(v_{0}, v_{1}, z_{j}^{*}: j \notin \mathcal{I}\right) .
$$

TheOREM 6.1. The mean-variance model $(\Delta, E, V)$ is generic if and only if all admissible minors of the matrix $C^{\#}$ (equivalently $Y^{\#}, Z^{\#}$ or $Z_{1}^{\#}$ ) are nonzero.

Proof. For $n=1$ the theorem is obvious, all models are generic and the set of admissible minors is empty.

Assume that our model is $n$-dimensional, $n \geq 2$, and $\mathcal{I}$ and $\mathcal{J}$ are disjoint sets of indices with union $\mathcal{I} \cup \mathcal{J}$ containing $n-2$ elements. We consider two cases depending on whether $\mathcal{I}$ is empty or not.

Lemma 6.3. If $\mathcal{I}=\emptyset$, then the vanishing of the $d_{\mathcal{I}, \mathcal{J}}$ minor of the matrix $C^{\#}$ (equivalently $Y^{\#}, Z^{\#}$ or $Z_{1}^{\#}$ ) is equivalent to the equality of the value of the mean $E$ on the two vertices not in $\mathcal{J}$.

Proof. Let $\mathcal{J}=\left(j_{1}, \ldots, j_{n-2}\right), j_{1}<\ldots<j_{n-2}$, and $k, l, k<l$, be the two missing indices. We have

$$
\begin{aligned}
d_{\emptyset, \mathcal{J}}\left(C^{\#}\right) & =\operatorname{det}\left(e, \widehat{\mu}, e_{j_{1}}, \ldots, e_{j_{n-2}}\right)=(-1)^{k+l+1} \operatorname{det}\left(\begin{array}{cc}
1 & \widehat{\mu}_{k} \\
1 & \widehat{\mu}_{l}
\end{array}\right) \\
& =(-1)^{k+l+1}\left(\widehat{\mu}_{l}-\widehat{\mu}_{k}\right) .
\end{aligned}
$$

Therefore the vanishing of the minor is equivalent to the equality

$$
E\left(z_{k}\right)=\widehat{\mu}_{k}=\widehat{\mu}_{l}=E\left(z_{l}\right) .
$$

Next assume that $\mathcal{I}$ is not empty. Let

$$
H_{1}=H\left(z_{i}: i \in \mathcal{I}\right), \quad H_{2}=H\left(z_{i}: i \notin \mathcal{J}\right) .
$$

Obviously $H_{1}$ is a subspace of $H_{2}$ of codimension 2 .

LEMma 6.4. If $\mathcal{I} \neq \emptyset$, then the following conditions are equivalent:

(i) The $d_{\mathcal{I}, \mathcal{J}}$ minor of the matrix $Z_{1}^{\#}$ (equivalently $Y^{\#}, Z^{\#}$ or $C^{\#}$ ) is zero.

(ii) $\Sigma_{H_{2}}$ intersects $H_{1}$, or is parallel to it, or consists of one point.

Proof. From Corollary 6.2 we get

$$
\Sigma_{H_{2}}=H_{2} \cap \operatorname{Lin}\left(v_{0}, v_{1}, z_{j}^{*}: j \in \mathcal{J}\right) .
$$

Therefore if $\Sigma_{H_{2}}$ intersects $H_{1}$ or is parallel to it then the vectors $v_{0}, v_{1}, z_{j}^{*}$ $(j \in \mathcal{J}), z_{i}(i \in \mathcal{I})$ are linearly dependent and the $d_{\mathcal{I}, \mathcal{J}}$ minor of the matrix $Z_{1}^{\#}$ vanishes.

If $\Sigma_{H_{2}}$ degenerates to a point then the vectors $v_{0}, v_{1}$ and $z_{j}^{*}$ are linearly dependent, hence the minor vanishes as well. 
On the other hand if the $d_{\mathcal{I}, \mathcal{J}}$ minor of $Z_{1}^{\#}$ is zero then there are coefficients $c_{0}, c_{1}, a_{i}(i \in \mathcal{I}), b_{j}(j \in \mathcal{J})$, not all zero, such that

$$
c_{0} v_{0}+c_{1} v_{1}+\sum_{i \in \mathcal{I}} a_{i} z_{i}+\sum_{j \in \mathcal{J}} b_{j} z_{j}^{*}=0 .
$$

We put

$$
z=\sum_{i \in \mathcal{I}} a_{i} z_{i}, \quad a=\sum_{i \in \mathcal{I}} a_{i} .
$$

There are three possibilities:

- $z=0$,

- $z \neq 0$ but $a=0$,

- $a \neq 0$.

CASE 1. If $z=0$ then the vectors $v_{0}, v_{1}, z_{j}^{*}(j \in \mathcal{J})$ are linearly dependent, hence $\Sigma_{H_{2}}$ consists of one point.

CAsE 2. If $a=0$ then $z$ is parallel to $H_{1}$. But on the other hand it belongs to $\operatorname{Lin}\left(v_{0}, v_{1}, z_{j}^{*}: j \in \mathcal{J}\right)$. We show that $z$ is a leading vector of $\Sigma_{H_{2}}$, i.e.

$$
\Sigma_{H_{2}}=\{q\}+\operatorname{Lin}(z),
$$

where $q$ is any point from the line $\Sigma_{H_{2}}$. Indeed, $z$ is parallel to $H_{1}$ which is a subspace of $H_{2}$, hence $\{q\}+\operatorname{Lin}(z) \subset H_{2}$. Next since $q, z \in \operatorname{Lin}\left(v_{0}, v_{1}, z_{j}^{*}\right.$ : $j \in \mathcal{J})$,

$$
\{q\}+\operatorname{Lin}(z) \subset H_{2} \cap \operatorname{Lin}\left(v_{0}, v_{1}, z_{j}^{*}: j \in \mathcal{J}\right)=\Sigma_{H_{2}} .
$$

Since $z \neq 0$ and $z$ is parallel to $H_{1}$, we conclude that $\Sigma_{H_{2}}$ is parallel to $H_{1}$.

CASE 3. If $a \neq 0$ we may put $\bar{z}=(1 / a) z$. Then $\bar{z} \in H_{1}$. But also $\bar{z} \in \operatorname{Lin}\left(v_{0}, v_{1}, z_{j}^{*}: j \in \mathcal{J}\right)$. Since $H_{1} \subset H_{2}$, we get

$z \in H_{1} \cap \operatorname{Lin}\left(v_{0}, v_{1}, z_{j}^{*}: j \in \mathcal{J}\right)=H_{1} \cap H_{2} \cap \operatorname{Lin}\left(v_{0}, v_{1}, z_{j}^{*}: j \in \mathcal{J}\right)=H_{1} \cap \Sigma_{H_{2}}$, which means that $H_{1}$ and $\Sigma_{H_{2}}$ have a nonempty intersection.

To finish the proof of the theorem one has to apply the above lemmas to all admissible minors. Note that the degeneration of $\Sigma_{H_{2}}$ to a point, when $\operatorname{dim} H>0$, is equivalent to the constancy of the mean $E$ on the space $H_{2}$, which is not possible for generic models.

Corollary 6.3. The set $\mathcal{M}_{\mathrm{g}}$ of $n$-dimensional generic models is an open dense semialgebraic subset of the set $\mathcal{M}$ of all $n$-dimensional models.

Proof. $\mathcal{M}_{\mathrm{g}}$ is the complement of an algebraic set described by vanishing of admissible minors.

COROLlary 6.4. The signs of admissible minors are invariants of the equivalence relation between generic models with fixed ordering of the vertices of $\Delta$. 
Proof. Admissible minors are continuous functions on the space of all generic models of a given dimension and with fixed ordering of the vertices. Since they are nonvanishing, their signs are constant on connected subsets of this space, in particular on the images of the unit interval.

The signs of admissible minors cannot be chosen arbitrarily. There are some restrictions. For example the determinant of the matrix

$$
C^{*}=\left(\begin{array}{ccc}
0 & 0 & e^{T} \\
0 & 0 & \widehat{\mu}^{T} \\
e & \widehat{\mu} & \widehat{C}
\end{array}\right)
$$

is positive (compare Lemma 3.1). But taking twice the Laplace expansion (compare $[3, \S 1.6])$ we obtain

$$
\operatorname{det} C^{*}=\sum d_{\mathcal{I}, \emptyset}\left(C^{\#}\right) d_{\emptyset, \mathcal{I}}\left(C^{\#}\right),
$$

where the sum extends over all sets $\mathcal{I}$ of indices with $\# \mathcal{I}=n-2$. Since at least one summand must be positive, we get:

Corollary 6.5. If $n \geq 3$ then for at least one set $\mathcal{I}$ with $\# \mathcal{I}=n-2$, the minors $d_{\mathcal{I}, \emptyset}$ and $d_{\emptyset, \mathcal{I}}$ have the same sign.

Basing on the characterization of generic models by means of admissible minors we get the following practical rule of checking the equivalence.

Corollary 6.6. Two generic models $(\Delta, E, V)$ and $\left(\Delta^{\prime}, E^{\prime}, V^{\prime}\right)$ are equivalent if there exists a continuous mapping

$$
\Phi:[0,1] \rightarrow \mathbb{R}^{N}, \quad t \mapsto\left(Z_{t}, \mu_{t}, C_{t}\right),
$$

such that:

(i) for all $t, \operatorname{det} Z_{t} \neq 0, \operatorname{det} C_{t}>0$ and $C_{t}$ is symmetric;

(ii) for every admissible minor $d_{\mathcal{I}, \mathcal{J}}$ and all $t, d_{\mathcal{I}, \mathcal{J}}\left(C_{t}^{\#}\right) \neq 0$, where $C_{t}^{\#}=\left(e, Z_{t}^{T} \mu_{t}, Z_{t}^{T} C_{t} Z_{t}\right.$, Id $)$;

(iii) $\Delta=\Delta\left(Z_{0} e_{1}, \ldots, Z_{0} e_{n}\right), E(x)=\mu_{0} x, V(x)=x^{T} C_{0} x$ for $x \in \mathbb{R}^{n}$;

(iv) $\Delta^{\prime}=\Delta\left(Z_{1} e_{1}, \ldots, Z_{1} e_{n}\right), E^{\prime}(x)=\mu_{1} x, V^{\prime}(x)=x^{T} C_{1} x$ for $x \in \mathbb{R}^{n}$.

LEMma 6.5. If $(\Delta, E, V)$ is a generic model, $\Delta=\Delta\left(e_{1}, \ldots, e_{n}\right), E(x)=$ $\mu^{T} x$ and $V(x)=x^{T} C x$ for $x \in \mathbb{R}^{n}$, then for any reals $a_{1}, a_{2}, b, c_{1}, c_{2}, c_{3}$ with $a_{i}>0, c_{i} \geq 0$ the model $\left(\Delta, E^{\prime}, V^{\prime}\right)$, where

$$
\begin{aligned}
& E^{\prime}(x)=a_{1} \mu^{T} x+b e^{T} x, \\
& V^{\prime}(x)=a_{2} x^{T} C x+c_{1} x^{T} e e^{T} x+c_{2} x^{T} \mu \mu^{T} x+c_{3} x^{T}(e+\mu)\left(e^{T}+\mu^{T}\right) x,
\end{aligned}
$$

for $x \in \mathbb{R}^{n}$, is equivalent to $(\Delta, E, V)$.

Proof. Since $c_{i} \geq 0$ and $a_{2}>0$, the new variance $V^{\prime}$ is positive definite. Multiplication of $\mu$ by a positive constant $a_{1}$ induces multiplication of all 
admissible minors by $a_{1}$. Analogously multiplication of $C$ by $a_{2}$ induces multiplication of admissible minors by a power of $a_{2}$. The other transformations do not change the admissible minors. Therefore the mapping

$$
\begin{gathered}
\Phi:[0,1] \rightarrow \mathbb{R}^{N}, \\
\Phi(t)=\left(\operatorname{Id},\left(1-t+t a_{1}\right) \mu+t b e,\right. \\
\left.\left(1-t+t a_{2}\right) C+t\left(c_{1} e e^{T}+c_{2} \mu \mu^{T}+c_{3}(e+\mu)\left(e^{T}+\mu^{T}\right)\right)\right),
\end{gathered}
$$

induces the equivalence.

Before finishing this section we show one more application of Corollary 6.6. Let $\operatorname{diag}\left(\sigma_{1}^{2}, \ldots, \sigma_{n}^{2}\right)$ denotes the diagonal $n \times n$ matrix with $\sigma_{i}^{2}$ 's on the diagonal. We shall consider the general noncorrelated rates of return model $(\Delta, E, V)$, where

$$
\Delta=\Delta\left(e_{1}, \ldots, e_{n}\right), \quad E(x)=\mu^{T} x, V(x)=x^{T} \operatorname{diag}\left(\sigma_{1}^{2}, \ldots, \sigma_{n}^{2}\right) x, \quad x \in \mathbb{R}^{n} .
$$

LEMMA 6.6. If the $\sigma_{i}$ are positive and $\mu_{i}$ are pairwise distinct then the general noncorrelated rates of return model

$$
\left(\Delta\left(e_{1}, \ldots, e_{n}\right), \mu^{T} x, x^{T} \operatorname{diag}\left(\sigma_{1}^{2}, \ldots, \sigma_{n}^{2}\right) x\right)
$$

is generic and is equivalent to the model from Example 3.1,

$$
\left(\Delta\left(e_{1}, \ldots, e_{n}\right), \mu^{T} x, x^{T} x\right) .
$$

Proof. Multiplying the columns of $C=\operatorname{diag}\left(\sigma_{1}^{2}, \ldots, \sigma_{n}^{2}\right)$ by positive constants we do not change the signs of the admissible minors $d_{\mathcal{I}, \mathcal{J}}\left(C^{\#}\right)$. Therefore the mapping

$$
\begin{gathered}
\Phi:[0,1] \rightarrow \mathbb{R}^{N}, \\
\Phi(t)=\left(\operatorname{Id}, \mu, \operatorname{diag}\left(\frac{\sigma_{1}^{2}}{1-t+t \sigma_{1}^{2}}, \ldots, \frac{\sigma_{n}^{2}}{1-t+t \sigma_{n}^{2}}\right)\right),
\end{gathered}
$$

induces the equivalence.

7. Three-dimensional models. In this section we give a complete classification of the three-dimensional generic models.

For simplicity we assume that the simplex $\Delta$ is spanned by the unit vectors $e_{i}, \Delta=\Delta\left(e_{1}, e_{2}, e_{3}\right)$, and $\mu_{1}<\mu_{2}<\mu_{3}$. In this case the signs of the minors $d_{\emptyset, \mathcal{J}}\left(C^{\#}\right)$ are fixed:

$$
\begin{aligned}
& d_{\emptyset,\{1\}}=\mu_{3}-\mu_{2}>0, \\
& d_{\emptyset,\{2\}}=\mu_{1}-\mu_{3}<0, \\
& d_{\emptyset,\{3\}}=\mu_{2}-\mu_{1}>0 .
\end{aligned}
$$

Therefore we have to deal only with the minors $d_{\{1\}, \emptyset}\left(C^{\#}\right), d_{\{2\}, \emptyset}\left(C^{\#}\right)$ and $d_{\{3\}, \emptyset}\left(C^{\#}\right)$. Due to Corollary 6.5 only 7 of the 8 possible combinations of 
signs may occur. The case $(-,+,-)$, i.e. $d_{\{1\}, \emptyset}<0, d_{\{2\}, \emptyset}>0, d_{\{3\}, \emptyset}<0$, is not possible. We show that this is the only restriction.

We remark that in the three-dimensional case the sign of $d_{\{i\}, \emptyset}\left(C^{\#}\right)$ is determined by the sign of the last $y$-coordinate of the $i$ th vertex (in the coordinates introduced in $\S 3$ ). Indeed, due to Lemma 6.1,

$$
\begin{gathered}
d_{\{i\}, \emptyset}\left(C^{\#}\right)=\sqrt{\delta} \operatorname{det} Y \cdot d_{\{i\}, \emptyset}\left(Y^{\#}\right) . \\
Y^{\#}=\left(\begin{array}{cccc}
1 & 0 & & \\
0 & 1 & Y & \left(Y^{-1}\right)^{T} \\
0 & 0 &
\end{array}\right), \quad Y=\left(\begin{array}{lll}
y_{1,0} & y_{2,0} & y_{3,0} \\
y_{1,1} & y_{2,1} & y_{3,1} \\
y_{1,2} & y_{2,2} & y_{3,2}
\end{array}\right),
\end{gathered}
$$

where

$$
e_{i}=y_{i, 0} v_{0}+y_{i, 1} v_{1}+y_{i, 2} v_{2}
$$

Hence

$$
d_{\{i\}, \emptyset}\left(C^{\#}\right)=\sqrt{\delta} \operatorname{det} Y \cdot y_{i, 2} .
$$

Moreover

$$
y_{i, 0}=\frac{1}{\sqrt{\gamma}}, \quad y_{i, 1}=\frac{\sqrt{\gamma}}{\sqrt{\delta}}\left(\mu_{i}-\frac{\beta}{\gamma}\right) .
$$

Therefore the simplest way to construct examples is to provide the $Y$ matrices. The only condition to check is

$$
\operatorname{det} Y>0 \text {. }
$$

ExAmples. We fix $\beta=0$ and $\alpha=\gamma=\delta=1$. Then $\mu=Y^{T} e_{2}$ and $C=Y^{T} Y$. In the table opposite we list representatives for each combination of signs of the admissible minors of $C^{\#}$. In the last column we also list all open subsimplexes of $\Delta$ crossed by the polygonal line $\Sigma_{\Delta}$ of relative minimal variance, i.e. the successive values of the function $\operatorname{sim}(t)$. In Figures 1 and 2 we show the triangles $\Delta$ in $\left(y_{1}, y_{2}\right)$-coordinates. Looking at the intersection of $\Delta$ and the lines $y_{1}=$ const we get the points of relative minimal variance.

Next we show that every generic three-dimensional model is equivalent to one from the above list.

TheOREM 7.1. Any three-dimensional models having the same signs of the admissible minors are equivalent.

Proof. First we order the vertices with respect to the expected value of return:

$$
\mu_{i, 1}<\mu_{i, 2}<\mu_{i, 3}, \quad i=0,1 .
$$

Next we deform continuously both models to models based on the same simplex spanned by unit vectors $e_{j}$. Due to Lemma 6.5 we may further 


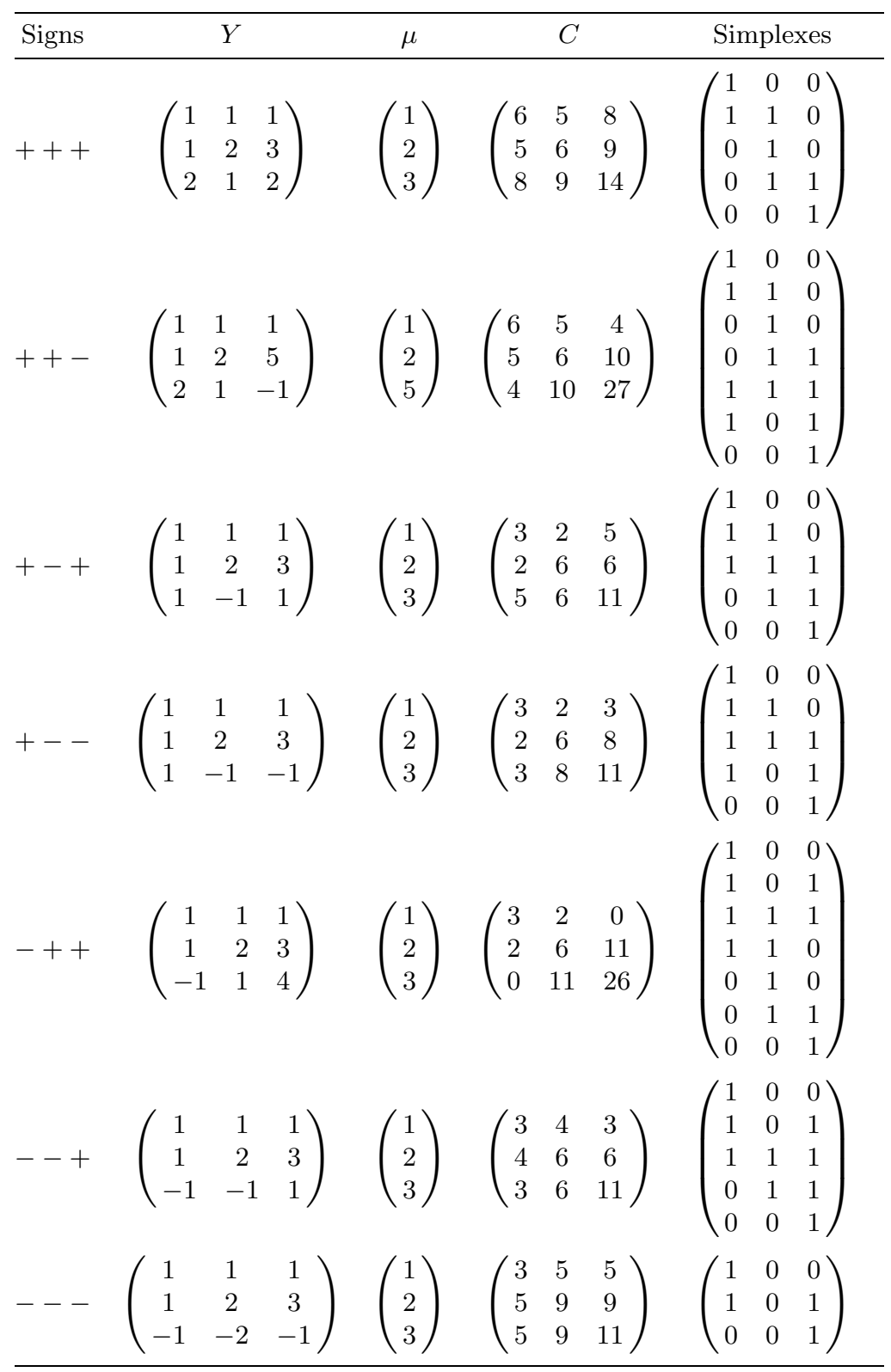

deform our models to equivalent ones having $\mu_{0,1}=\mu_{1,1}=-1, \mu_{0,2}=\mu_{1,2}$ $=0$ and $\mu_{0,3}>0, \mu_{1,3}>0$. We may assume that $\mu_{0,3} \leq \mu_{1,3}$.

If $\mu_{0,3}=\mu_{1,3}$ (i.e. $E_{0}=E_{1}=E$ ) then our models $\left(\Delta, E, V_{0}\right)$ and $\left(\Delta, E, V_{1}\right)$ can be joined by a linear path $\left(\Delta, E,(1-t) V_{0}+t V_{1}\right), t \in[0,1]$, which induces the equivalence. Indeed, since the admissible minors $d_{\{i\}, \emptyset}$ depend linearly on $t$, there is no change of sign. 

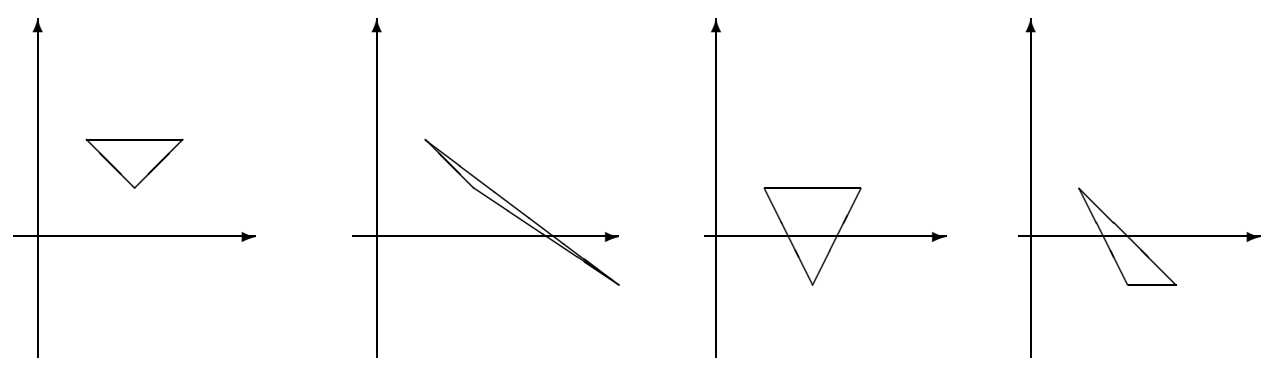

Fig. 1. Cases $(+++),(++-),(+-+)$ and $(+--)$
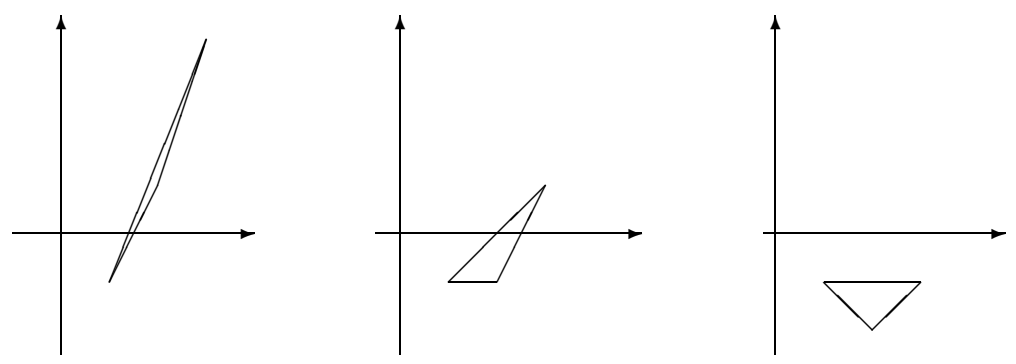

Fig. 2. Cases $(-++),(--+)$ and $(---)$

If $\mu_{0,3}<\mu_{1,3}$ we transform $\left(\Delta, E_{0}, V_{0}\right)$ to an equivalent model $\left(\Delta, E_{1}, V^{\prime}\right)$. Let $C$ be the matrix of $V_{0}$. We multiply the first row and first column of $C$ by $\mu_{1,3} / t$ and the second row and second column by $\left(\mu_{1,3}+1\right)(t+1)$. We denote the resulting matrix by $C_{t}$. Moreover we put $\mu_{t}=(-1,0, t)^{T}$. The mapping

$$
\Phi(t)=\left(\mathrm{Id}, \mu_{t}, C_{t}\right) \quad \text { for } t \in\left[\mu_{0,3}, \mu_{1,3}\right]
$$

induces the equivalence. To finish the proof we apply the line equivalence described above.

8. One-factor models. The one-factor models describe markets with one dominant synthetic asset which determines the returns of all other assets (Sharpe-Lintner version of CAPM, see [7, pp. 148-149], or APT [7, p. 159]). Namely, such models base on the assumption that there exist a random variable $r$ and constants $\beta_{i}$ and $r_{f}$ such that the rate of return of the $i$ th asset satisfies

$$
r_{i}=r_{f}+\beta_{i} r+\varepsilon_{i},
$$

where $\varepsilon_{i}$ 's are not correlated among themselves and not correlated with $r$. Most often $r_{f}$ coincides with the risk free return. Let $m=E(r), s^{2}=V(r)$ and $\sigma_{i}^{2}=V\left(\varepsilon_{i}\right)$. Then we get the following mean-variance model:

$$
\mu=r_{f} e+m \beta, \quad C=s^{2} \beta \beta^{T}+\operatorname{diag}\left(\sigma_{1}^{2}, \ldots, \sigma_{n}^{2}\right) .
$$


THEOREM 8.1. If $m>0$ and $\beta_{i}$ are pairwise distinct then the one-factor model

$$
\left(\Delta\left(e_{1}, \ldots, e_{n}\right),\left(r_{f} e+m \beta\right)^{T} x, x^{T}\left(s^{2} \beta \beta^{T}+\operatorname{diag}\left(\sigma_{1}^{2}, \ldots, \sigma_{n}^{2}\right)\right) x\right)
$$

is generic and is equivalent to the noncorrelated rates of return model

$$
\left(\Delta\left(e_{1}, \ldots, e_{n}\right), \beta^{T} x, x^{T} x\right)
$$

Proof. Apply Lemmas 6.5 and 6.6.

Corollary 8.1. If $\beta_{1}<\ldots<\beta_{n}$ then the polygonal line $\Sigma_{\Delta}$ is a union of $2 n-3$ segments. The interior of the middle one is contained in the interior of $\Delta$, the interior of the ith, $i<n-1$, in the interior of $\Delta\left(e_{1}, \ldots, e_{i+1}\right)$, and the interior of the $j t h, j>n-1$, in the interior of $\Delta\left(e_{j+2-n}, \ldots, e_{n}\right)$.

Proof. Compare Example 5.1 and Theorem 5.1.

Note that since the equivalence of generic models is stable, i.e. it is preserved by small enough changes, the assertion of Corollary 8.1 remains valid also if our model is only close enough to a one-factor model.

\section{References}

[1] Z. Bodie and R. C. Merton, Finance, Prentice-Hall, 2000.

[2] R. Korn and E. Korn, Option Pricing and Portfolio Optimization. Modern Methods of Financial Mathematics, Amer. Math. Soc., 2001.

[3] A. Kurosh, Higher Algebra, Mir, Moscow, 1972.

[4] H. M. Markowitz, Porfolio selection, J. Finance 7 (1952), 77-91.

[5] - Mean-Variance Analysis in Portfolio Choice and Capital Markets, Blackwell, 1992.

[6] F. K. Reilly and K. C. Brown, Investment Analysis and Portfolio Management, Dryden Press, 1977.

[7] S. Wang and Y. Xia, Portfolio Selection and Asset Pricing, Springer, 2002.

Institute of Mathematics

Warsaw University

Banacha 2

02-097 Warszawa, Poland

E-mail: jwptxa@mimuw.edu.pl

Received on 4.6.2001;

revised version on 27.1.2003 